\title{
EP NEWS AND INNOVATIONS
}

\author{
CE Mark Approval for Magnetic Resonance Conditional Labeling of its Quadra Allure MP Cardiac Resynchronization \\ Therapy Pacemaker \\ People worldwide are afflicted with congestive heart failure and new cases are diagnosed worldwide each year. \\ Studies have shown that cardiac resynchronization therapy (CRT) can improve the quality of life for many patients \\ with heart failure, a progressive condition in which the heart weakens and loses its ability to pump an adequate supply \\ of blood. CRT resynchronizes the lower chambers (ventricles) of the heart by sending uniquely programmed electrical \\ impulses to stimulate each ventricle to beat in sync for optimal cardiac performance.
}

The Quadra Allure MP CRT-P, with its first-to-market MultiPoint ${ }^{\mathrm{TM}}$ Pacing technology, now offers patients the flexibility to undergo full-body diagnostic magnetic resonance imaging (MRI) scans of up to 1.5 Tesla (measurement for the strength of a magnetic field) for other conditions.

The Quadra Allure MP CRT-P with MRI labeling is designed to work with the Quartet ${ }^{\mathrm{TM}}$ LV lead, which has four electrodes to offer maximum flexibility for different pacing configurations to help manage heart failure patients. Despite advances in quadripolar pacing technology, non-responders to CRT therapy continue to be a clinical concern. The MultiPoint Pacing technology has been shown to enhance patients' response to CRT. The technology allows physicians the opportunity to capture more left ventricular tissue quickly by delivering pacing pulses to multiple left ventricle locations rather than the traditional single pulse for each heartbeat.

Multiple clinical trials, including a recent study, demonstrate that MultiPoint Pacing technology may be particularly beneficial in patients not responding to traditional bi-ventricular pacing therapy, which accounts for approximately one third of the total population of patients receiving CRT. With more options to treat patients who don't respond to CRT, the clinician can reduce the rate of CRT non-responders and reduce the likelihood of costly lead revision through a second intervention procedure.

The new Quadra Allure MP CRT-P with MRI labeling is also compatible with the SyncAV ${ }^{\mathrm{Tm}}$ CRT software, designed to build upon the MultiPoint Pacing technology and further optimize the comprehensive CRT portfolio. The SyncAV CRT technology, which automatically adjusts pacing based on real-time changes in a patient's cardiac condition, also provides physicians the opportunity to further improve treatment of patients who have responded positively to traditional CRT. Quadripolar technology offers physicians more options to manage heart failure and facilitates additional pacing configurations within the heart that offer physicians options not available in traditional bipolar systems. While the Quadra Allure MP CRT-P with MultiPoint Pacing and SyncAV CRT technology is approved in the U.S., work continues toward approval for MR-conditional labeling.

\section{First and Only 42 Joule Ultra-High Energy ICD for Heart Failure Patients}

Patients suffering from tachycardia an implantable cardioverter defibrillator (ICD) is necessary to convert dangerous arrhythmias to a normal heart rhythm. ICDs provide life-saving shocks to the heart as needed. Patient anatomy and health conditions impact the heart's response to shock therapy. To save lives, it is critical for an ICD to deliver an effectively powerful shock as soon as possible.

The launch of Inventra HF-T, the first and only implantable cardioverter defibrillator (ICD) available in the United States that delivers an "ultra-high" energy with 42 joules (J) on the first shock. Inventra is an ICD designed for heart failure patients known as a cardiac resynchronization therapy defibrillator (CRT-D).

The implant has a lifespan of up to 7.5 years, depending on usage, is compatible with full body scanning under certain conditions, and features monitoring algorithms designed to reduce the number of unnecessary shocks.

Initial U.S. Commercial Performance Of The WATCHMAN ${ }^{\mathrm{TM}}$ Left Atrial Appendage Closure Device

Left atrial appendage closure (LAAC) is a treatment strategy to reduce the risk of left atrial appendage blood clots from entering the bloodstream and causing a stroke in patients with non-valvular atrial fibrillation (AF). 
U.S. commercial performance results of the WATCHMAN ${ }^{\mathrm{TM}}$ Left Atrial Appendage Closure (LAAC) have shown data demonstrated a high rate of procedural success and low rate of complications for the device which offers stroke risk reduction for patients with non-valvular atrial fibrillation (AF) who are seeking an alternative to long-term warfarin therapy.

Procedural data were collected via WATCHMAN ${ }^{\mathrm{TM}}$ clinical specialists during 3,822 consecutive LAAC procedures performed between March 2015 and May 2016 by 382 operating physicians at 169 U.S. medical centers. In this case series, there was a $95.6 \%$ implant success rate with a median procedure time of 50 minutes.

Half of the procedures were performed by new implanting physicians without previous experience with the device. The overall rate of complications evaluated within these data was low at $1.63 \%$, and compared favorably to the clinical trial data leading to device approval, validating the rigorous process for selecting and training new operators. Pericardial tamponade requiring intervention was the most frequent major procedural complication, which was seen in $1.02 \%$ of patients. In an additional $0.29 \%$ of patients, a hemodynamically insignificant pericardial effusion was observed that did not require intervention. Device embolization, procedure-related stroke and mortality rates also remained low at $0.24 \%, 0.08 \%$ and $0.08 \%$, respectively.

The formal national registry capturing data on left atrial appendage occlusion (LAAO) procedures - the LAAO Registry was approved in August, 2016.

\section{Largest Observational Study to Date of AMPLATZER Amulet Left Atrial Appendage Occluder Demonstrates High} Implant Success and Strong Safety Profile

During atrial fibrillation (AF), the heart's upper chambers (atria) beat erratically and out of sync with the two lower chambers, resulting in poor blood flow. The LAA is a tube-shaped appendage connected to the left atrium of the heart that can potentially hold static blood during an episode of $\mathrm{AF}$, increasing the likelihood of clot formation. Research shows that in AF patients, approximately 90 percent of all cardiac blood clots form in the LAA. If a clot forms in the LAA and is then released into the heart, it may enter blood circulation, travel to the brain, block a vessel and cause an ischemic stroke. The current standard of care to treat ischemic stroke in AF patients is blood-thinning medications, which comes with major, sometimes fatal, bleeding risks.

According to the World Health Organization (WHO), an estimated 15 million strokes occur worldwide each year. In 2010, stroke cost the United States an estimated $\$ 53.9$ billion in health care services, medications and missed days of work.

AMPLATZER $^{\mathrm{TM}}$ Amulet $^{\mathrm{TM}}$ device is used to completely seal off the left atrial appendage (LAA) in patients diagnosed with non-valvular atrial fibrillation (AF). Sealing off the LAA in patients with AF may prevent the risk of blood clot formation and release, potentially reducing that risk.

The prospective, multicenter, international AMPLATZER Amulet Observational Study of LAA occlusion (closure) enrolled 1,073 patients at 64 centers across 18 countries. Initial results from the study showed a 98.8 percent implant success rate adding to the clinical evidence that the AMPLATZER ${ }^{\mathrm{TM}}$ Amulet $^{\mathrm{TM}}$ LAA Occluder is a safe alternative for prevention of stroke in patients with non-valvular atrial fibrillation (AF).

Other key findings of the Amulet Observational study included: 1.) Closure rate was 99 percent at one to three month follow-up visit $(\mathrm{n}=364)$. Closure was defined as absence of flow or flow of $<3 \mathrm{~mm}$ jet into the LAA. All echocardiography results were adjudicated by an independent core lab. 2.) Acute device/procedure related major adverse events within seven days post procedure occurred in 2.7 percent (29) of 1,073 patients.

The AMPLATZER ${ }^{\mathrm{TM}}$ Amulet $^{\mathrm{TM}}$ Left Atrial Appendage Occluder is CE Mark approved, but remains an investigational device in the United States. The shape and size of an LAA can vary greatly. The AMPLATZER ${ }^{\mathrm{TM}}$ Amulet $^{\mathrm{TM}}$ LAA Occluder has a conformable disc and lobe designed for complete occlusion of the LAA and offers the broadest size range available. The device offers physicians options to help meet the anatomical demands of patients in need of LAA closure.

\section{MHLW approval in Japan of EnSite Precision Cardiac Mapping System}

Physicians use catheter ablation to treat arrhythmias, and diagnostic catheters record electrical information from the heart and display it in a three-dimensional anatomical model, which is used to study the abnormal rhythm.

Another catheter is used for the actual ablation. The physician positions the ablation catheter so it lies on or very close to the targeted tissue. The small area of heart tissue under the tip of the ablation catheter is heated by high-frequency energy, creating a lesion. As a result, this tissue is no longer capable of conducting or sustaining the arrhythmia. 
MHLW approval of EnSite Precision ${ }^{\mathrm{TM}}$ Cardiac Mapping System and a Sensor Enabled ${ }^{\mathrm{TM}}$ Mapping Catheter constitutes introduction of an entirely new suite of technologies including innovations in hardware, catheters, surface electrodes (patches) and software features designed for improved ease of use. The new EnSite Precision ${ }^{\mathrm{TM}}$ Cardiac Mapping System is designed to provide automation, flexibility and precision in cardiac mapping during the treatments of patients with cardiac arrhythmias. Prominent European electrophysiology labs and hospitals have embraced this next generation platform, which is now installed and active in more than 100 sites across Europe and has been used in thousands of cases in Europe since receiving CE Mark in January 2016.

In addition to the approval of EnSite Precision ${ }^{\mathrm{TM}}$ Cardiac Mapping System, there has been secured approval for the TactiCath $^{\mathrm{TM}}$ Quartz Irrigated Ablation Catheter, which is designed to provide physicians with more control to modify that force during ablation procedures in order to create more effective lesions for patients being treated for atrial fibrillation (AF). Combined, these two approvals will allow physician partners across Japan to better treat patients with $\mathrm{AF}$ and cardiac arrhythmias in this critical global market.

Cardiac mapping provides a live view of the heart so physicians can visualize and navigate cardiac anatomy to deliver more precise ablation therapy. The new EnSite Precision ${ }^{\mathrm{TM}}$ Cardiac Mapping System offers a new dual technology platform that provides highly detailed anatomical models and maps to enable more efficient treatment of a wide range of arrhythmias - including complex arrhythmias, such as atrial fibrillation or ventricular tachycardia.

The EnSite Precision Cardiac Mapping System is flexible and enhances workflow efficiency by allowing physicians to optimize mapping of the heart chambers using the new Sensor Enabled toolset and customize procedures to address the circumstances of each case. Designed for advanced handling and maneuverability, the Advisor ${ }^{\mathrm{TM}}$ FL Circular Mapping Catheter, Sensor Enabled ${ }^{\mathrm{TM}}$ allows for precise navigation and model creation with impedance-field flexibility and magnetic-field stability.

This cardiac mapping system also allows real-time catheter navigation to occur using minimal fluoroscopy, thus reducing potential for risks associated with excessive radiation exposure for patients and clinicians.

The EnSite Precision ${ }^{\mathrm{TM}}$ Cardiac Mapping System is based on the Medical EnSite ${ }^{\mathrm{TM}}$ Velocity ${ }^{\mathrm{TM}}$ Cardiac Mapping System. This introduces new solutions, including the EnSite ${ }^{\mathrm{TM}}$ AutoMap Module, which will enable the electrophysiologist to more quickly perform morphology matching that identifies the source of the irregular heartbeat using automated cardiac rhythm mapping tools to facilitate treatment. The EnSite ${ }^{\mathrm{TM}}$ AutoMap Module includes the new TurboMap feature, which will allow the physician to build a map of the heart 10 times faster than current systems using recorded data.

\section{Approval for the cryoICE ${ }^{\mathrm{TM}}$ Platform in Japan}

Atrial fibrillation affects more than 33 million people worldwide. Treatments for atrial fibrillation (Afib) and left atrial appendage (LAA) management are critical issues. The Japanese Ministry of Health, Labor and Welfare (MHLW) has approved the cryoICE ${ }^{\mathrm{TM}}$ platform and CRYO2 cryoablation probe. The approval marks the first time that these products will be available in the Japanese market.

This new approval will offer leading solutions to cardiac surgeons and Japanese patients for the treatment of atrial fibrillation.

\section{Landmark Stroke Data from the RESPECT Trial}

The RESPECT trial final results demonstrated that after long-term follow-up (average of $\sim 6$ years), treatment with the AMPLATZER $^{\mathrm{TM}}$ PFO Occluder was more effective than medical therapy alone in reducing the risk for recurrent ischemic stroke by 45 percent.

RESPECT, a clinical trial of nearly 1,000 patients diagnosed with patent foramen ovale (PFO), which is a small opening between the upper chambers of the heart, and cryptogenic stroke (stroke from an unknown cause), compared PFO closure to medical therapy alone.

The AMPLATZER ${ }^{\mathrm{TM}}$ PFO Occluder is designed to lower the risk of stroke by sealing off the hole between the left and right atria - the patent foramen ovale. Normally the foramen ovale, which is a small opening between the left and right atria, closes shortly after birth, but remains open in approximately 25 percent of adults. Left untreated, a PFO could allow dangerous clots from the veins to pass from the right side of the heart to the left and travel up to the brain and potentially cause a stroke. 
These final analyses which include additional follow-up beyond those on which FDA approval was based, support continued patient benefit over an average of approximately six years of follow-up. The AMPLATZER ${ }^{\mathrm{TM}}$ PFO Occluder was recently approved by the Food and Drug Administration and is the only device approved in the United States for PFO closure to reduce the risk of recurrent ischemic stroke in patients, predominantly between the ages of 18 and 60 years, who have had a cryptogenic stroke due to a presumed paradoxical embolism, as determined by a neurologist and cardiologist following an evaluation to exclude known causes of ischemic stroke.

\section{FDA approves new device for prevention of recurrent strokes in certain patients}

The U.S. Food and Drug Administration approved the Amplatzer ${ }^{\mathrm{TM}}$ PFO Occluder device. The PFO Occluder reduces the risk of a stroke in patients who previously had a stroke believed to be caused by a blood clot that passed through a small hole in the heart, called a patent foramen ovale (PFO), and then traveled to the brain.

About 25 to 30 percent of Americans have a PFO, which typically causes no health problems and does not require treatment. The cause of most strokes can be identified, such as poorly controlled high blood pressure, narrowed blood vessels due to cholesterol deposits and scar tissue (atherosclerosis), or a blood clot caused by an abnormal heart rhythm (atrial fibrillation). However, in some patients, medical tests cannot identify the cause of the stroke, which is referred to as a cryptogenic stroke. In a small percentage of these patients, it is believed that the PFO provided a path for a blood clot to travel to the brain where it blocked a blood vessel resulting in a stroke. Patients with a cryptogenic stroke and a PFO may be at an increased risk of having a second stroke.

The AMPLATZER ${ }^{\mathrm{TM}}$ PFO Occluder is inserted through a catheter that is placed in a leg vein and advanced to the heart. It is then implanted close to the hole in the heart between the top right chamber (right atrium) and the top left chamber (left atrium). The device had been on the market more than a decade ago under a humanitarian device exemption (HDE), but was voluntarily withdrawn by the manufacturer in 2006 after the FDA concluded that the target population for this device was greater than 4,000 patients and that the device no longer qualified for an HDE approval. For the past 10 years, no FDA-approved heart occluder devices have been on the market specifically indicated to close PFOs to reduce the risk of a recurrent stroke in patients with a prior cryptogenic stroke.

In approving the AMPLATZER ${ }^{\mathrm{TM}}$ PFO Occluder, the FDA concluded that the device demonstrated a reasonable assurance of safety and effectiveness. The safety and efficacy was assessed in a randomized study that evaluated 499 participants aged 18 to 60 years old who were treated with the Amplatzer PFO Occluder plus blood-thinning medications compared to 481 participants who were treated with blood-thinning medications alone. While the rate of new strokes in both treatment groups was very low, the study found a 50 percent reduction in the rate of new strokes in participants using the AMPLATZER ${ }^{\mathrm{TM}}$ PFO Occluder plus blood-thinning medications compared to participants taking only blood-thinning medications.

Adverse effects associated with the device or the implantation procedure include injury to the heart, irregular and/or rapid heart rate (atrial fibrillation), blood clots in the heart, leg or lung, bleeding and stroke.

The AMPLATZER ${ }^{\mathrm{TM}}$ PFO Occluder device should not be used in patients with a heart valve infection or other untreated infections, or a heart tumor or blood clot at the implant site. The device is also contraindicated in patients with other abnormal connections between the heart chambers or in whom the cardiovascular anatomy or blood clots would interfere with the ability to move the catheter used to deliver the device to the heart.

\section{The Unique Ability of PURE EP System to Visualize High Frequency Signals Highlighted}

Ventricular fibrillation (VF), ventricular tachycardia (VT) and atrial fibrillation (AF) are a few conditions referred to as an arrhythmia. AF is the most widespread, affecting approximately 33 million people worldwide, and 6.1 million in the U.S. according to the Centers for Disease Control and Prevention (CDC).

There is a well-documented correlation between $\mathrm{AF}$ and stroke and other associated risks, such as heart failure. Research shows that catheter ablation therapy for treatment of arrhythmia patients is still not a perfect science. New electrophysiology (EP) device technologies, like PURE $\mathrm{EP}^{\mathrm{TM}}$ System, are needed to improve this procedure's success rates. Many of today's EP systems have limited dynamic range, which translates into problems when amplifying small cardiac signals in order to see them, ultimately distorting and saturating large signals. Electrical noise is also an issue, which makes it difficult for clinicians to differentiate real physiologic signals from noise. It is with the mission of solving these and other issues that the development of PURE EP ${ }^{\mathrm{TM}}$ System, a technology that is poised to capture a significant portion of the rapidly growing EP market.

PURE EP ${ }^{\mathrm{TM}}$ System is a surface electrocardiogram and intracardiac multichannel signal acquisition and analysis system engineered to assist electrophysiologists in making clinical decisions in real-time by acquiring and displaying 
high-fidelity cardiac signal recordings and providing clarity of data which may be used to guide the electrophysiologists in identifying ablation targets - areas of tissue to treat that otherwise create a heart rhythm disturbance (arrhythmia).

\section{PURE EP System to Enter Seventh Pre-Clinical Trial}

PURE EP ${ }^{\mathrm{TM}}$ System is a surface electrocardiogram (ECG) and intracardiac multichannel recording and analysis system developed to assist electrophysiologists in making clinical decisions in real-time, albeit diagnostic or during an ablation procedure.

The PURE EP ${ }^{\mathrm{TM}}$ System has the potential to shorten procedure time and reduce the necessity for repeated procedures that are frequent with current devices.

Many of today's EP systems have limited dynamic range, which translates into problems in needing to amplify small signals in order to see them, which ultimately distorts resolution and saturates large signals. Electrical noise is also an issue, which makes it difficult for clinicians to differentiate real physiologic signals from noise. PURE EP ${ }^{\mathrm{TM}}$ System has shown effective in helping to solve these issues.

Because the PURE $\mathrm{EP}^{\mathrm{TM}}$ qualifies with the FDA as a Class II medical device, extensive clinical trials are not required. 